\title{
Revista Colombiana de

\section{Prevalencia y asociación de factores de riesgo cardiovascular en hombres con disfunción eréctil}

\section{Carolina Sandoval-Salinas ${ }^{a, *}$, Juan M. Martínez ${ }^{a}$, Héctor A. Corredor ${ }^{a}$, José P. Saffon ${ }^{a}$, Claudia Jaramillo ${ }^{\mathrm{b}}$ y Fernán Mendoza ${ }^{\mathrm{b}}$}

a Grupo de Investigación Clínica, Boston Medical Group. Bogotá, Colombia

b Fundación Clínica Shaio, Bogotá, Colombia

Recibido el 22 de julio de 2019; aceptado el 17 de noviembre de 2019

Disponible en Internet el 10 de octubre de 2020

\author{
PALABRAS CLAVE \\ Disfunción eréctil; \\ Prevalencia; \\ Factores de riesgo \\ cardiovascular; \\ Enfermedad \\ cardiovascular
}

\begin{abstract}
Resumen
Objetivo: estimar la prevalencia de los factores de riesgo para enfermedad cardiovascular en hombres con disfunción eréctil atendidos en un centro especializado en diferentes ciudades de Colombia y evaluar su asociación con el grado de severidad de la enfermedad.

Métodos: estudio de corte transversal analítico. Se revisaron los registros clínicos de pacientes con diagnóstico de disfunción eréctil que fueron atendidos durante el primer semestre del año 2018. Se analizaron las características sociodemográficas y clínicas de los pacientes, y se estimó la prevalencia de los factores de riesgo cardiovascular. Se exploró la asociación entre estos factores y el grado de severidad de la disfunción eréctil por medio de modelos multivariados logísticos ordinales.

Resultados: se incluyeron 2.484 pacientes que cumplieron los criterios de selección, con una edad promedio de 48,7 años $( \pm 14,1)$. 2.112 sujetos presentaron uno o más factores de riesgo cardiovascular, para una prevalencia global de $85,2 \%$. La prevalencia estimada para factor evaluado fue la siguiente: sobrepeso y obesidad $70,9 \%$, edad superior a 45 años $60,0 \%$, hiperlipidemia $25,1 \%$, hipertensión $21,4 \%$, consumo de tabaco $17,7 \%$, diabetes $16,7 \%$, infarto agudo de miocardio $2,21 \%$ y antecedente de alguna enfermedad cardiovascular diferente a infarto 1,68\%. En el análisis multivariado se observó que la diabetes y la edad son factores que aumentan el riesgo de severidad de la disfunción eréctil.
\end{abstract}

\footnotetext{
* Autor para correspondencia.

Correo electrónico: csandoval@bostonmedical.com.co (C. Sandoval-Salinas).
} 


\section{KEYWORDS}

Erectile dysfunction;

Prevalence;

Cardiovascular risk

factors;

Cardiovascular

disease
Conclusiones: es alta la prevalencia de factores de riesgo cardiovascular en hombres colombianos con disfunción eréctil. En ese sentido, el sobrepeso u obesidad es el factor modificable más frecuente en esta población. El grado de disfunción puede estar asociado con la edad y la diabetes.

(c) 2020 Sociedad Colombiana de Cardiología y Cirugía Cardiovascular. Publicado por Elsevier España, S.L.U. Este es un artículo Open Access bajo la licencia CC BY-NC-ND (http:// creativecommons.org/licenses/by-nc-nd/4.0/).

\section{Prevalence and association with cardiovascular risk in men with erectile dysfunction}

\begin{abstract}
Objective: To estimate the prevalence of risk factors of cardiovascular disease in men with erectile dysfunction attending a specialist clinic in different cities in Colombia, as well as their association with the level of severity of the disorder.

Methods: A cross-sectional analytical study was conducted using a review of the medical records of patients diagnosed with erectile dysfunction and seen during the first six months of 2018. An analysis was made of the socio-demographic and clinical characteristics of the patients, and the prevalence of cardiovascular risk factors was calculated. The association between these factors and the level of severity of the erectile dysfunction was determined using ordinal multivariate logistic models.

Results: The study included a total of 2,484 patients that met the selection criteria. The mean age was 48.7 ( \pm 14.1 ) years. The majority $(2.112)$ of subjects had one or more cardiovascular risk factors, with an overall prevalence of $85.2 \%$. The estimated prevalence for a factor evaluated was as follows: overweight and obesity; $70.9 \%$, age greater than 45 years; $60.0 \%$, hyperlipidaemia; $25.1 \%$, hypertension; $21.4 \%$, smoking; $17.7 \%$, diabetes; $16.7 \%$, acute myocardial infarction; $2.21 \%$, and a history of any cardiovascular disease other than an infarction; $1.68 \%$. In the multivariate analysis it was observed that diabetes and age are factors that increase the risk of severity of erectile dysfunction.

Conclusions: The prevalence of cardiovascular risk factors is high in Colombian men with erectile dysfunction. As regards this, overweight or obesity is the most common modifiable factor in this population. The level of dysfunction may be associated with age and diabetes.

(c) 2020 Sociedad Colombiana de Cardiología y Cirugía Cardiovascular. Published by Elsevier España, S.L.U. This is an open access article under the CC BY-NC-ND license (http:// creativecommons.org/licenses/by-nc-nd/4.0/).
\end{abstract}

\section{Introducción}

La disfunción eréctil es la incapacidad persistente de conseguir y mantener una erección suficiente que permita una relación sexual satisfactoria ${ }^{1}$. Es una condición relacionada con aspectos orgánicos o psicológicos que tiene un impacto significativo en la calidad de vida de las personas afectadas y de su pareja. En Colombia se ha estimado que aproximadamente el $52,8 \%$ de los hombres mayores de 40 años presentan algún grado de disfunción ${ }^{2}$.

Es clara la relación que hay entre los factores que aumentan el riesgo cardiovascular y la presencia de disfunción; evidencia reciente muestra cómo la disfunción eréctil puede ser una manifestación temprana de enfermedad coronaria y enfermedad vascular periférica ${ }^{3,4}$, por lo cual esta condición debería considerarse como una señal de peligro potencial de la enfermedad cardiovascular.

Entre los factores cardiovasculares modificables se ha evidenciado que en hombres fumadores la prevalencia de disfunción llega hasta el $40 \%$ frente a un $28 \%$ en la población general ${ }^{5}$; así mismo, se ha observado una relación entre la exposición pasiva al cigarrillo y la incidencia de disfunción eréctil ${ }^{5}$ y entre el consumo de cigarrillo y la reducción en la frecuencia de las erecciones matutinas ${ }^{6}$. Por otra parte, en hombres obesos el riesgo de disfunción es $60 \%$ más, comparado con hombres de peso dentro de rangos normales ${ }^{7}$. Respecto a los factores controlables, se ha demostrado la asociación con hipertensión ${ }^{7,8}$, diabetes ${ }^{2,7,9}$, hiperlipidemia ${ }^{10}$ y síndrome metabólico ${ }^{10}$.

Pese a las diferentes publicaciones sobre el tema en varios países, actualmente no se cuenta con información local para conocer la situación de estos pacientes en Colombia, de manera que reconociendo cuáles son los factores de riesgo más prevalentes puedan plantearse acciones para mitigar el problema. El objetivo de este estudio es estimar la prevalencia de factores de riesgo cardiovascular en pacientes con disfunción eréctil en Colombia para el primer semestre del año 2018 y explorar la asociación entre estos factores y la severidad de la disfunción eréctil.

\section{Materiales y métodos}

Se llevó a cabo un estudio observacional de corte transversal analítico. El tamaño de muestra se estimó a partir del total 
de pacientes con disfunción eréctil que asistieron a consulta a una clínica especializada en salud sexual masculina con sedes en diferentes ciudades del país durante el primer semestre del año $2018(n=2.613$ hombres), esperando una frecuencia entre el $20 \%$ y $50 \%$ de los factores de riesgo cardiovascular según datos reportados en la literatura ${ }^{11,12}$, con una precisión absoluta del $3 \%$ alrededor del estimador y un nivel de confianza del 95\%; así, el tamaño de muestra estimado fue de 758 pacientes. No obstante, teniendo en cuenta que la información se obtuvo de registros históricos, se incluyó a todos los sujetos de la población de interés, que cumplieron los criterios de selección.

Para la recolección de la información se extrajeron de forma automática los datos de las historias clínicas en medio digital, incluyendo pacientes mayores de 18 años con diagnóstico clínico de disfunción eréctil y puntaje de IIEF-EF inferior a 22, que fueron atendidos entre el $1^{\circ}$. de enero y el 30 de junio de 2018. Se excluyeron pacientes con disfunción de origen psicológico o con datos incompletos para los análisis de interés. Las variables evaluadas fueron: edad, estimada a partir de la fecha de nacimiento del paciente; índice de masa corporal, calculado a partir de la talla reportada en la cédula del paciente, y el peso registrado en consulta con básculas de diferentes marcas según la ciudad de atención y el año de adquisición, y calibradas de acuerdo con el estándar de calidad institucional; tensión arterial medida con tensiómetros digitales de diferentes marcas según la ciudad de atención y el año de adquisición, y calibrados de acuerdo con el estándar de calidad institucional; consumo de alcohol; consumo de tabaco; consumo de drogas recreativas; puntaje del cuestionario IIEF-EF; puntaje de dureza de la erección -EHS - y antecedentes médicos de otros factores de riesgo cardiovascular, como diabetes, hipertensión, hiperlipidemias, enfermedad cardiovascular e infarto agudo del miocardio, reportados por el paciente. El diagnóstico inicial de diabetes e hiperlipidemia registrado en el examen clínico fue confirmado con los resultados de las pruebas de laboratorio, tomadas y procesadas en un laboratorio clínico externo con sedes en las diferentes ciudades donde se incluyeron pacientes y procesos estandarizados, en los casos en los que se contaba con esta información.

Para el control de calidad de la extracción de la información se revisó aleatoriamente el $5 \%$ de las historias clínicas incluidas. Los datos incongruentes o valores extremos fueron verificados en la historia clínica original por dos investigadores diferentes de quien realizó la selección inicial de los pacientes.

Se analizaron las características sociodemográficas y clínicas de los sujetos de investigación usando herramientas de la estadística descriptiva: para las variables categóricas se estimaron frecuencias absolutas y relativas, y para las variables numéricas se estimaron medidas de tendencia central y de dispersión, dependiendo de la distribución de los datos. Se estimó la prevalencia de los factores de riesgo cardiovascular de forma global y por categorías de acuerdo con el grado de severidad de la disfunción, y se hizo una exploración de la asociación entre estas variables usando pruebas de $x^{2}$ para variables categóricas y ordinales, considerando valores de significación del $5 \%$ y pruebas a dos colas para las hipótesis planteadas.

Por último, se desarrolló un modelo de regresión logística multinomial para cuantificar el grado de asociación entre cada factor de riesgo cardiovascular y el grado de disfunción eréctil ajustando por la presencia de otros factores de riesgo y variables confusoras. Para esto se planteó un modelo inicial que incluyó todos los factores de riesgo cardiovascular, independiente de su valor de $\mathrm{p}$ en el análisis bivariado y otras variables con un valor de $p<0,2$ en ese mismo análisis.

Tabla 1 Características de la población de estudio

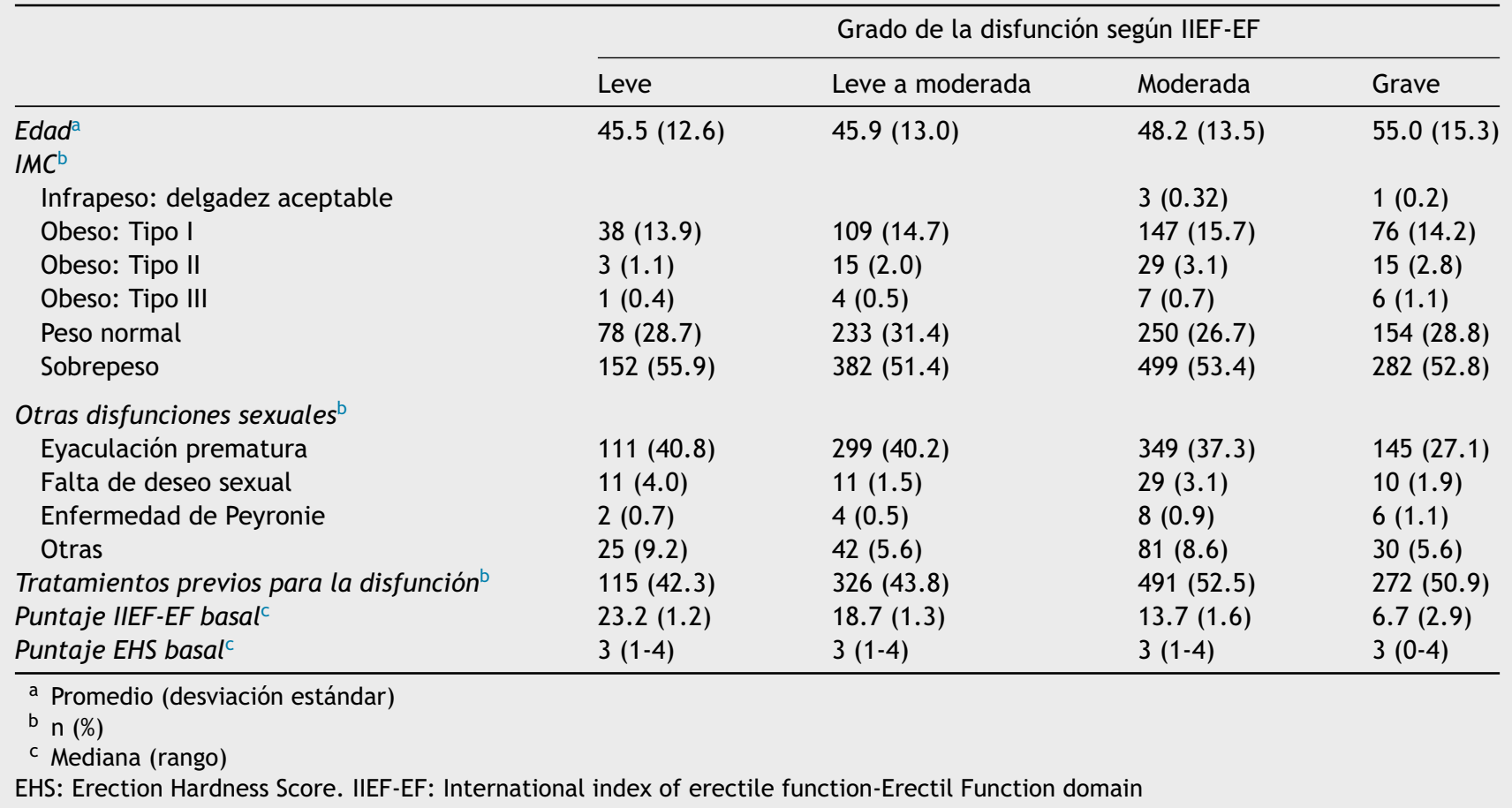




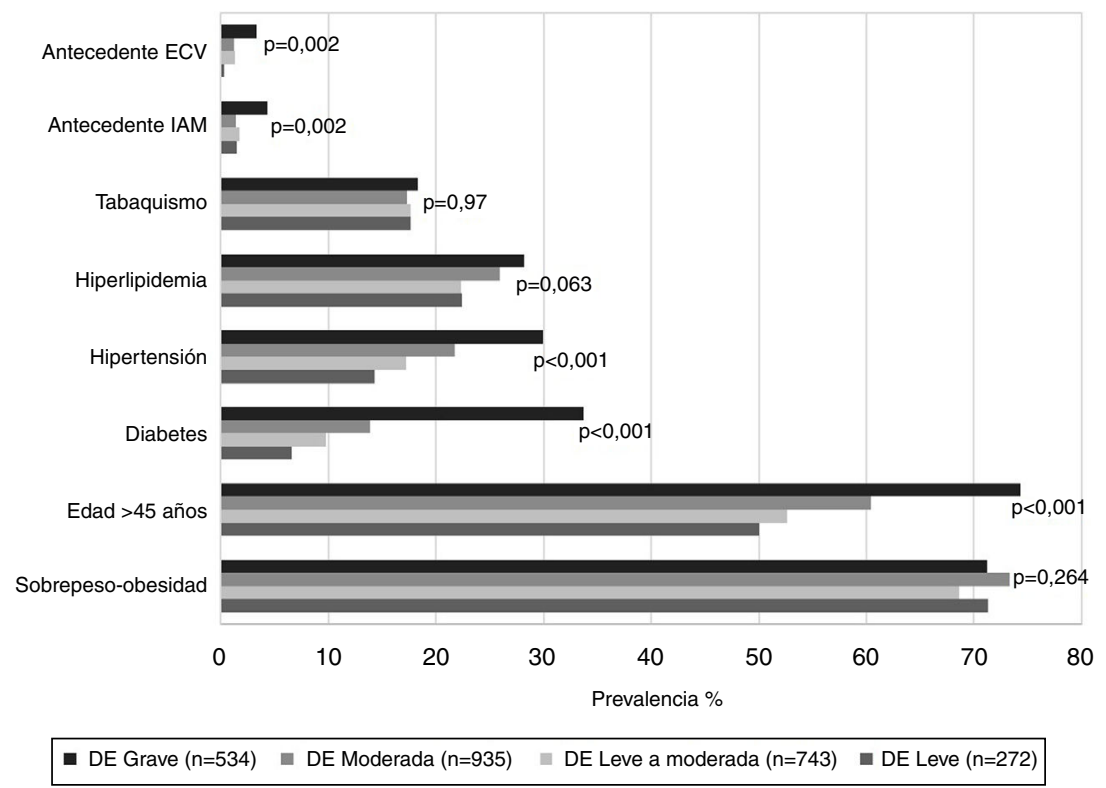

Figura 1 Prevalencia de factores de riesgo cardiovascular según el grado de severidad de la disfunción eréctil DE: disfunción eréctil; ECV: enfermedad cardiovascular; IAM: infarto agudo del miocardio.

Para la estimación de la prevalencia y el análisis bivariado y multivariado, la edad se categorizó como menor de 45 años e igual o mayor a 45 años, y según el IMC se categorizó a los pacientes como normo-peso $(I M C<25)$ o sobrepeso u obesidad (IMC $\geq 25)$. Los análisis se hicieron con Stata 15.1 (College Station, TX: StataCorp LLC).

\section{Resultados}

Durante el periodo de análisis fueron atendidos 2.613 pacientes con diagnóstico de disfunción eréctil; 2.484 de ellos cumplieron los criterios de selección y fueron incluidos en el estudio. La mayoría de los pacientes residían en Bogotá $(34,6 \%, n=861)$, Medellín $(9,54 \%, n=237)$ y Cali $(6.60 \%, n=164)$. La edad media fue 48,7 años $( \pm 14,1)$ y el valor promedio del índice de masa corporal (IMC) fue 27,2 $( \pm 3,7)$ con solo el $28,7 \%$ de los pacientes en peso normal (tabla 1).

En cuanto a la disfunción eréctil, el promedio del puntaje del módulo de función eréctil de la escala IIEF-15 (IIEF-EF) fue 14,7 puntos $( \pm 5,5)$, observándose que la mayor proporción de pacientes tenían disfunción eréctil moderada $(37,6 \%)$ o leve a moderada $(29,9 \%)$, con base en este mismo cuestionario. La mediana del puntaje de dureza de la erección (Erection Hardness Score-EHS) fue 3 puntos $(I R Q=1)$. $40,6 \%$ de los pacientes tenían otra disfunción sexual además de la impotencia; entre ellas la más frecuente fue la eyaculación precoz $(36,4 \%) ; 48,5 \%$ de los sujetos de investigación habían tomado medicamentos para la disfunción previo al ingreso a la clínica (tabla 1).

Por otro lado, 2.112 los sujetos presentaron uno o más factores de riesgo cardiovascular, para una prevalencia global de 85,1\% (IC 95\% 83,5-86,4\%). De forma más específica, las prevalencias de los factores de riesgo cardiovascular evaluados fueron: sobrepeso y obesidad $70,9 \%$ (IC 95\% 69,1-72,7\%), edad superior a 45 años 60,0\% (IC
95\% 58,0-61,9\%), hiperlipidemia 25,0\% (IC 95\% 23,3-26,7\%), hipertensión $21,3 \%$ (IC 95\%, 19,7-23,0\%), consumo de tabaco 17,7\% (IC 95\% 16,1-19,2\%), diabetes 16,1\% (IC 95\% 14,7$17,6 \%)$, antecedente de infarto agudo del miocardio $2,2 \%$ (IC 95\% 1,6-2,8\%) y antecedente de alguna enfermedad cardiovascular diferente a infarto 1,6\% (IC 95\% 1,2-2,2\%). La prevalencia de estos factores por grado de severidad de la disfunción se presenta en la figura 1.

En el análisis bivariado se encontró que ser mayor de 45 años, tener diabetes y tener hipertensión está asociado con padecer disfunción moderada o grave; así mismo, se encontró asociación entre disfunción eréctil grave y haber tenido infarto agudo del miocardio o un antecedente de enfermedad cardiovascular diferente a infarto. Sin embargo, al ajustar la estimación por otros factores o variables confusoras en el análisis multivariado se observó, que comparado con tener enfermedad leve, el riesgo de tener enfermedad moderada o grave aumenta en hombres diabéticos (OR 1,95 IC 95\% 1,15-3,3 y OR 5,57 IC 95\% 3,28-9,43, respectivamente), así como aumenta el riesgo de enfermedad grave en hombres mayores de 45 años (OR 1,76 IC 95\% 1,25-2,47) (tabla 2).

\section{Discusión}

La disfunción eréctil es una enfermedad prevalente que afecta la calidad de vida de los hombres y sus parejas. En diferentes estudios se ha evaluado su relación con enfermedad cardiovascular y se ha encontrado que ambas, además de estar relacionadas, comparten factores de riesgo similares $^{4,13}$. En general, se considera tan fuerte la correlación entre los factores de riesgo de la disfunción eréctil y la enfermedad cardiovascular, que en algunos estudios, como el desarrollado por Dong et al., se sugiere que la disfunción es probablemente un factor de riesgo independiente para enfermedad cardiovascular ${ }^{4}$. 
Tabla 2 Asociación entre factores de riesgo cardiovascular y severidad de la disfunción eréctil según IIEF-EF, análisis crudo y ajustado

\begin{tabular}{|c|c|c|c|c|c|c|}
\hline \multirow[t]{2}{*}{ Severidad DE según IIEF-EF } & \multicolumn{3}{|c|}{ Análisis crudo } & \multicolumn{3}{|c|}{ Análisis multivariado } \\
\hline & OR & valor de $\mathrm{p}$ & IC 95\% & OR & valor de $p$ & IC 95\% \\
\hline \multicolumn{7}{|c|}{ Leve (categoría de comparación) } \\
\hline \multicolumn{7}{|c|}{ Leve a moderada } \\
\hline$>45$ años & 1,09 & 0,524 & 0,83 a 1,45 & 1 & 0,985 & 0,74 a 1,35 \\
\hline Diabetes & 1,54 & 0,115 & 0,9 a 2,63 & 1,5 & 0,15 & 0,86 a 2,59 \\
\hline Hiperlipidemias & 1 & 0,977 & 0,71 a 1,39 & 0,98 & 0,89 & 0,69 a 1,38 \\
\hline Hipertensión arterial & 1,24 & 0,272 & 0,84 a 1,83 & 1,18 & 0,44 & 0,78 a 1,79 \\
\hline $\mathrm{IAM} / \mathrm{ECV}$ & 1,63 & 0,329 & 0,61 a 4,35 & 1,46 & 0,46 & 0,54 a 3,97 \\
\hline Sobrepeso - obesidad & 0,87 & 0,389 & 0,64 a 1,19 & 0,85 & 0,31 & 0,62 a 1,16 \\
\hline Tabaquismo & 1 & 0,995 & 0,69 a 1,44 & 1,05 & 0,803 & 0,72 a 1,53 \\
\hline Alcohol & 0,82 & 0,163 & 0,62 a 1,08 & 0,81 & 0,155 & 0,6 a 1,08 \\
\hline Tratamiento previo & 1,07 & 0,649 & 0,81 a 1,41 & 1,09 & 0,565 & 0,82 a 1,45 \\
\hline \multicolumn{7}{|l|}{ Moderada } \\
\hline$>45$ años & 1,5 & 0,003 & 1,15 a 1,97 & 1,23 & 0,167 & 0,92 a 1,66 \\
\hline Diabetes & 2,28 & 0,002 & 1,36 a 3,81 & 1,95 & 0,013 & 1,15 a 3,3 \\
\hline Hiperlipidemias & 1,21 & 0,234 & 0,88 a 1,67 & 1,06 & 0,746 & 0,76 a 1,47 \\
\hline Hipertensión arterial & 1,66 & 0,008 & 1,14 a 2,41 & 1,33 & 0,159 & 0,89 a 1,99 \\
\hline $\mathrm{IAM} / \mathrm{ECV}$ & 1,41 & 0,492 & 0,53 a 3,72 & 1,03 & 0,95 & 0,38 a 2,79 \\
\hline Sobrepeso - obesidad & 1,08 & 0,599 & 0,8 a 1,46 & 0,97 & 0,836 & 0,71 a 1,32 \\
\hline Tabaquismo & 0,98 & 0,902 & 0,69 a 1,39 & 1 & 0,994 & 0,69 a 1,44 \\
\hline Alcohol & 0,87 & 0,323 & 0,67 a 1,14 & 0,83 & 0,202 & 0,62 a 1,1 \\
\hline Tratamiento previo & 1,51 & 0,003 & 1,15 a 1,98 & 1,47 & 0,007 & 1,11 a 1,94 \\
\hline \multicolumn{7}{|l|}{ Severa } \\
\hline$>45$ años & 2,86 & $<0,001$ & 2,1 a 3,88 & 1,76 & 0,001 & 1,25 a 2,47 \\
\hline Diabetes & 7,18 & $<0,001$ & 4,31 a 11,96 & 5,57 & $<0,001$ & 3,28 a 9,43 \\
\hline Hiperlipidemias & 1,36 & 0,075 & 0,97 a 1,92 & 0,99 & 0,97 & 0,69 a 1,43 \\
\hline Hipertensión arterial & 2,56 & $<0,001$ & 1,74 a 3,76 & 1,42 & 0,106 & 0,93 a 2,17 \\
\hline $\mathrm{IAM} / \mathrm{ECV}$ & 4,21 & 0,003 & 1,64 a 10,8 & 2,25 & 0,105 & 0,85 a 5,97 \\
\hline Sobrepeso - obesidad & 1,05 & 0,806 & 0,72 a 1,54 & 1,13 & 0,547 & 0,75 a 1,71 \\
\hline Tabaquismo & 0,97 & 0,874 & 0,71 a 1,34 & 0,79 & 0,165 & 0,56 a 1,1 \\
\hline Alcohol & 0,59 & $<0,001$ & 0,44 a 0,79 & 0,56 & $<0,001$ & 0,41 a 0,77 \\
\hline Tratamiento previo & 1,42 & 0,02 & 1,06 a 1,9 & 1,36 & 0,05 & 1 a 1,85 \\
\hline
\end{tabular}

DE: Disfunción eréctil; HTA: Hipertensión arterial; IIEF-EF: International index of erectile function-Erectil Function domain; OR: odds ratio

En la actualidad se recomiendan algoritmos para el manejo de la disfunción eréctil en pacientes con o sin enfermedad cardiovascular conocida ${ }^{14}$; así mismo, está recomendada la evaluación de los factores de riesgo cardiovascular en estos pacientes, de tal manera que se puedan implementar modificaciones en el estilo de vida para el manejo de la enfermedad y se identifiquen aquellos sujetos que realmente se benefician de una valoración por un servicio de cardiología ${ }^{15}$.

Los resultados de este estudio muestran la alta prevalencia de algunos factores de riesgo compartidos con la enfermedad cardiovascular en hombres colombianos con disfunción eréctil, para los cuales se ha documentado previamente una relación en otras poblaciones ${ }^{4,13}$. Comparado con estudios realizados en hombres estadounidenses ${ }^{16,17}$, las prevalencias estimadas en nuestro estudio para factores como hiperlipidemia o hipertensión son inferiores, así como también difiere la asociación entre el grado de la disfunción eréctil y estos factores, la cual ha sido documentada en esta misma población ${ }^{8}$. Esto puede ser explicado por diferencias en la alimentación o en el estilo de vida entre las poblaciones, lo que se evidencia en la prevalencia estimada en la población general ${ }^{18,19}$.

Un hallazgo importante de este estudio es evidenciar que uno de los factores más prevalentes en nuestra población son el sobrepeso y la obesidad. A pesar de mostrar tendencias de aumento en todo el mundo, el sobrepeso y la obesidad son factores de riesgo modificables, lo cual permite plantear intervenciones para reducir su prevalencia en la población, disminuyendo así, no solo el riesgo de disfunción eréctil grave sino también el riesgo de morbilidad y mortalidad por enfermedad cardiovascular y por otras enfermedades asociadas a esta condición ${ }^{20}$.

Aunque este estudio tiene las limitaciones propias de los estudios retrospectivos, como el posible sesgo de información por falta de un registro riguroso en las historias clínicas de todos los datos de los pacientes o la falta de evaluación de otras variables confusoras de interés, vale la pena recalcar 
la importancia de ser información local, que resulta fundamental para el desarrollo de políticas y la toma de decisiones clínicas más específicas para nuestra población, sobre todo al tratarse de algunos factores de riesgo cardiovascular que pueden ser modificables o controlables.

\section{Conclusiones}

La prevalencia de factores de riesgo cardiovascular en hombres colombianos con disfunción eréctil es alta, y, en ese sentido, el sobrepeso u obesidad constituyen el factor modificable más frecuente en esta población. El grado de disfunción puede estar asociado con la edad y la diabetes.

\section{Financiación}

El estudio fue financiado con recursos del centro de investigación clínica de Boston Medical Group y recursos propios de los autores.

\section{Conflicto de intereses}

Ninguno.

\section{Bibliografía}

1. NIH Consensus Conference. Impotence. NIH Consensus Development Panel on Impotence. JAMA. 1993;270:83-90.

2. Morillo L, Díaz J, Estevez E, Costa A, Méndez H, Dávila H, et al. Prevalence of erectile dysfunction in Colombia Ecuador, and Venezuela: a population-based study (DENSA). Int J Impot Res. 2002:10-8.

3. Gandaglia G, Briganti A, Jackson G, Kloner RA, Montorsi F, Montorsi P, et al. A Systematic review of the association between erectile dysfunction and cardiovascular disease. Eur Urol. 2014;65:968-78.

4. Dong J-Y, Zhang Y-H, Qin L-Q. Erectile dysfunction and risk of cardiovascular disease: meta-analysis of prospective cohort studies. J Am Coll Cardiol. 201;58(13):1378-85.

5. Tengs TO, Osgood ND. The link between smoking and impotence: two decades of evidence. Prev Med (Baltim). 2001;32:447-52.

6. Hirshkowitz M, Karacan I, Howell JW, Arcasoy MO, Williams RL. Nocturnal penile tumescence in cigarette smokers with erectile dysfunction. Urology. 1992;39:101-7.
7. Saigal CS, Wessells H, Pace J, Schonlau M, Wilt TJ. Urologic Diseases in America Project. Predictors and prevalence of erectile dysfunction in a racially diverse population. Arch Intern Med. 2006;166:207.

8. Sun P, Swindle R. Are men with erectile dysfunction more likely to have hypertension than men without erectile dysfunction? A naturalistic national cohort study. J Urol. 2005;174:244-8.

9. Binmoammar TA, Hassounah S, Alsaad S, Rawaf S, Majeed A. The impact of poor glycaemic control on the prevalence of erectile dysfunction in men with type 2 diabetes mellitus: a systematic review. JRSM Open. 2016;7, 205427041562260.

10. Roumeguère $T$, Wespes $E$, Carpentier $Y$, Hoffmann $P$, Schulman CC. Erectile dysfunction is associated with a high prevalence of hyperlipidemia and coronary heart disease risk. Eur Urol. 2003;44:355-9.

11. Chang ST, Chu CM, Hsu JT, Lin PC, Shee JJ. Surveillance of cardiovascular risk factors for outpatients in different erectile dysfunction severity. Int J Impot Res. 2009;21:116-21.

12. Ryan JG, Gajraj J. Erectile dysfunction and its association with metabolic syndrome and endothelial function among patients with type 2 diabetes mellitus. J Diabetes Complications. 2012;26:141-7.

13. Montorsi P, Ravagnani PM, Galli S, Rotatori F, Veglia F, Briganti $A$, et al. Association between erectile dysfunction and coronary artery disease Role of coronary clinical presentation and extent of coronary vessels involvement: The COBRA trial. Eur Heart J. 2006;27:2632-9.

14. Vlachopoulos C, Jackson G, Stefanadis C, Montorsi P. Erectile dysfunction in the cardiovascular patient. Eur Heart J. 2013;34:2034-46.

15. Gandaglia G, Briganti A, Jackson G, Kloner RA, Montorsi F, Montorsi $\mathrm{P}$, et al. A systematic review of the association between erectile dysfunction and cardiovascular disease. Eur Urol. 2014;65:968-78.

16. Walczak MK, Lokhandwala N, Hodge MB, Guay AT. Prevalence of cardiovascular risk factors in erectile dysfunction. J Gend Specif Med. 2002;5:19-24.

17. Seftel A, Sun P, Swindle R. The prevalence of hypertension, hyperlipidemia, diabetes mellitus and depression in men with erectile dysfunction. J Urol. 2004;171:2341-5.

18. Fryar CD, Ostchega Y, Hales CM, Zhang G, Kruszon-Moran D. Hypertension prevalence and control among adults: United States, 2015-2016. NCHS data brief no 289. Hyattsville, MD: National Center for Health Statistics; 2017.

19. High Blood Pressure (Hypertension) Information | cdc.gov [Internet]. [Acceso 4 Jan 2019]. Disponible en: https://www.cdc.gov/bloodpressure/.

20. Hruby A, Hu FB. The epidemiology of obesity: a big picture. Pharmacoeconomics. 2015;33:673-89. 